\title{
Effect of fibulin-5 on aldosterone-induced apoptosis in human ascending aortic smooth muscle cells
}

\author{
YONGJI YAN $^{1 *}$, XUJIE LIU $^{2 *}$, XUANCHEN LI ${ }^{2 *}$, XUAN ZHOU ${ }^{2}$,

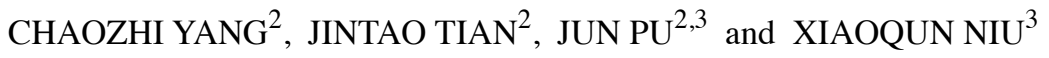 \\ ${ }^{1}$ Department of Urology, Beijing University of Chinese Medicine Dongzhimen Hospital, Beijing 100700; \\ Departments of ${ }^{2}$ Neurosurgery and ${ }^{3}$ Pneumology, Second Affiliated Hospital of Kunming Medical University, \\ Kunming, Yunnan 650101, P.R. China
}

Received September 9, 2017; Accepted December 1, 2017

DOI: $10.3892 / \mathrm{etm} .2021 .10328$

\begin{abstract}
The aim of the current study was to investigate the effect of aldosterone on apoptosis in human aortic smooth muscle cells (HA-VSMC) and to determine the role of fibulin-5 in the aldosterone-induced apoptosis of HA-VSMC cells. Through the construction of a fibulin-5 eukaryotic overexpression vector and a short hairpin RNA interference plasmid, fibulin-5 was overexpressed and silenced, respectively. The role of fibulin-5 in the aldosterone-induced apoptosis of HA-VSMC was subsequently determined. The overexpression of fibulin-5 inhibited the apoptosis of cells, particularly at low concentrations of aldosterone; a smaller effect on apoptosis was induced by high concentrations of aldosterone. fibulin- 5 knockdown promoted the apoptosis of cells induced by high concentrations of aldosterone but had a smaller effect on the apoptosis of cells induced by low concentrations of aldosterone. Therefore, the results of the current study indicate that fibulin-5 inhibits the aldosterone-induced apoptosis of HA-VSMC cells and that this effect may be altered by changing the aldosterone concentration.
\end{abstract}

\section{Introduction}

Aldosterone-producing adenoma (APA) is a benign adenoma that causes autonomous secretion of aldosterone (1). Its symptoms include fatigue; the prevalence of APA in patients with hypertension is $8 \%$ (1). Few patients die of APA and

Correspondence to: $\mathrm{Dr}$ Jun $\mathrm{Pu}$ or Professor Xiaoqun Niu, Department of Pneumology, Second Affiliated Hospital of Kunming Medical University, 374 Dianmian Avenue, Kunming, Yunnan 650101, P.R. China

E-mail: 1371001307@qq.com

E-mail: 673409256@qq.com

*Contributed equally

Key words: aldosterone, human aortic smooth muscle cells, apoptosis, fibulin-5 most cases of APA-associated deaths are caused by the complications, including hypertensive cerebral haemorrhage, arrhythmia caused by hypokalaemia, renal failure and electrolyte disturbance (1). The primary method for treating APA is surgical resection; however, poor regulation of blood pressure following surgery is a long-term clinical problem as hypertension can lead to vascular remodeling (2). If the surgery occurred after the vascular remodeling or medication did not fully treat hypertension, the rate of post-operative complications may increase (2). Aldosterone may induce vascular remodelling; it can directly lead to vascular remodelling and induce the apoptosis of vascular smooth muscle cells. The apoptosis of vascular endothelial cells and an imbalance in their proliferation may lead to vascular remodelling (3).

Fibulin-5 belongs to the fibulin family of proteins, which are extracellular matrix proteins and may serve an important role in aldosterone-induced vascular remodelling. Fibulin-5 is a multifunctional protein that serves an important role in maintaining the formation and stabilization of elastic fibres, as well as the proliferation, migration and apoptosis of human aortic vascular smooth muscle cells $(4,5)$. This experiment stimulates the vascular remodeling which occurs in PA. It also shows that the mice lack of fibulin-5 get more severe vascular remodeling and fibulin-5 can improve it (6). In the presence of aldosterone, fibulin-5 deposition in the rat aorta significantly increases, suggesting that fibulin-5 may serve an important role in aldosterone-induced vascular remodelling (7). Furthermore, the regulation of fibulin-5 during apoptosis differs in different types of cells or in different environments in the same cell (8-12). Therefore, it is important to clarify the effect of fibulin-5 on the apoptosis of human ascending aortic smooth muscle cells treated with aldosterone.

\section{Materials and methods}

Cells and reagents. Human ascending aortic smooth muscle cells (HA-VSMCs) were purchased from the Advanced Research Center, Central South University. Cells were cultured in complete medium [RPMI 1640 medium (Hyclone; GE Healthcare Life Sciences) containing 12\% fetal bovine serum (both Gibco; Thermo Fisher Scientific, Inc.)] at $37^{\circ} \mathrm{C}$. All cells were routinely resuscitated and passaged and cells 
used in the following experiment were all in the logarithmic growth phase.

Apoptosis-inducing agents, including cycloheximide, which acted as a positive control, and aldosterone were purchased from Sigma-Aldrich; Merck KGaA, dissolved in dimethyl sulfoxide, and stored at $4^{\circ} \mathrm{C}$. Puromycin was purchased from InvivoGen, dissolved in complete medium, sterilized using a $0.22 \mu \mathrm{m}$ filter and stored at $-20^{\circ} \mathrm{C}$. Lipofectamine ${ }^{\circledR} 2000$ was purchased from Invitrogen; Thermo Fisher Scientific, Inc. Fibulin-5 monoclonal antibody was purchased from Abcam. The Lenti-Pac ${ }^{\mathrm{TM}}$ HIV Expression Packaging kit, All-in-One ${ }^{\mathrm{TM}}$ First-Strand cDNA Synthesis kit and SYBR-Green Master with Rox kit were all purchased from GeneCopoeia, Inc.

Construction of interference and overexpression vectors. The interference and overexpression vectors were designed and constructed by GeneCopoeia, Inc. The fibulin-5 overexpressing vector was constructed using the OmicsLink ${ }^{\mathrm{TM}}$ expression cloning vector EX-Z5658-Lv105. The control plasmid used was EX-NEG-Lv105. The fibulin-5 gene (1,347 bps; NM_006329) was cloned into the vector, competent cells were transformed and the culture was expanded by selecting a colony and culture in LB liquid culture medium supplemented with $100 \mu \mathrm{g} / \mathrm{ml}$ ampicillin for $12-16 \mathrm{~h}$ at $37^{\circ} \mathrm{C}$ at $200 \mathrm{rpm}$. Extracted plasmids were sequenced for lentivirus packaging. The interference vector was constructed using the OmicsLink ${ }^{\mathrm{TM}}$ short hairpin (sh)RNA Expression Clone vector psi-LVRU6P. The fibulin-5 shRNA sequence was 5'-GGATACTCACTGTTACCATTC-3' and the scramble shRNA sequence of the control vector was 5'-GCTTCGCGCCGTAGTCTTA-3'.

Lentiviral packaging of the fibulin-5 interference and overexpression vector. Using the Lenti-Pac ${ }^{\mathrm{TM}}$ HIV Expression Packaging kit (GeneCopoeia), the interference plasmid and overexpression plasmid were transfected into $293 \mathrm{~T}$ cells with DNA-lipofectamine 2000 Reagent (Invitrogen; Thermo Fisher Scientific, Inc.). Complete medium was replaced $8 \mathrm{~h}$ after transfection. The culture supernatants rich in lentiviral particles were collected following $48 \mathrm{~h}$ culture and stored at $-80^{\circ} \mathrm{C}$.

Construction of the fibulin-5 stably transmitted HA-VSMC cell line. Following $72 \mathrm{~h}$ fibulin-5 interference, HA-VSMC cells were infected with overexpression and control lentivirus particles (MOI, 5) using polybrene ${ }^{\mathrm{TM}}$ (EMD Millipore; Merck $\mathrm{KGaA}$ ) and $1.5 \mu \mathrm{g} / \mathrm{ml}$ puromycin to kill any cells that were not successfully transfected. The medium was changed every 2 days and continuous screening continued for 2 weeks with complete medium to expand the cell culture. Reverse transcription-quantitative polymerase chain reaction (RT-qPCR) was used to verify the expression of fibulin-5 mRNA and western blotting was performed to evaluate the expression of fibulin-5 protein.

Detection of a stable transfection effect. RNA was extracted using TRIzol ${ }^{\circledR}$ (Thermo Fisher Scientific, Inc.) from each group of cells in the logarithmic growth phase. The concentration and purity of RNA were determined using a spectrophotometer at a wavelength of 260 and $280 \mathrm{~nm}$. RT was performed using the All-in-One ${ }^{\mathrm{TM}}$ First-Strand cDNA Synthesis kit (GeneCopoeia) under the following reaction conditions: $42^{\circ} \mathrm{C}$ for $60 \mathrm{~min}$ and $70^{\circ} \mathrm{C}$ for $5 \mathrm{~min}$. Primers were designed and synthesized by GeneCopoeia. qPCR was performed using the SYBR-Green Master with Rox kit (GeneCopoeia). The reaction conditions for qPCR were $95^{\circ} \mathrm{C}$ for $10 \mathrm{~min}$, followed by 40 cycles of $95^{\circ} \mathrm{C}$ for $15 \mathrm{sec}, 60^{\circ} \mathrm{C}$ for $30 \mathrm{sec}$ and $72^{\circ} \mathrm{C}$ for $30 \mathrm{sec}$. mRNA expression in each group was quantified using the $2^{-\Delta \Delta \mathrm{Cq}}$ method (13).

Western blotting. The expression level of fibulin-5 was detected in the human ascending aortic smooth muscle cells from the following groups by western blotting: HA-VSMC, mFibulin-5, mFibulin-5 Ctrl, shFibulin-5 Ctrl, and shFibulin-5. Briefly, protein samples were obtained using RIPA lysis buffer (Beyotime Institute of Biotechnology) containing protease inhibitor cocktail (EMD Millipore; Merck KGaA), following which protein concentrations were measured using Bicinchoninic Acid Protein Assay Kit (Beyotime Institute of Biotechnology). Samples at $30 \mathrm{mg} /$ lane were separated by $10 \%$ SDS-PAGE and then transferred onto a PVDF membrane (EMD Millipore; Merck KGaA). $\beta$-Actin was used as a loading control. The membrane was then blocked with $5 \%$ fat-free milk at room temperature for $40 \mathrm{~min}$ and separately with either mouse anti-Fibulin-5 (1:1,000; cat. no. ab66339; Abcam) or mouse anti- $\beta$-actin (1:5,000; cat. no. ab6276; Abcam) primary antibodies overnight at $4^{\circ} \mathrm{C}$. The membranes were then incubated with peroxidase-conjugated goat anti-mouse $\mathrm{IgG}$ secondary antibody at room temperature for $1 \mathrm{~h}$ (1:10,000; cat. no. A4416; Sigma-Aldrich; Merck KGaA). Protein bands were visualized using SuperSignal ${ }^{\mathrm{TM}}$ West Femto Maximum Sensitivity Substrate (Thermo Fisher Scientific, Inc). ImageJ software (version 1.46; National Institutes of Health) was used for grey scale analysis and to calculate relative protein expression.

Assessment of apoptosis in HA-VSMCs following treatment with aldosterone. HA-VSMC cells in the logarithmic growth phase were plated in a 6 -well plate at a density of $5 \times 10^{5}$. Following incubation, cells were treated with $0,1,10,100$ and $1000 \mu \mathrm{M}$ aldosterone for 1, 2, 3, 4 and 5 days. Cells were then collected. Cells treated with $4 \mathrm{mg} / \mathrm{l}$ actinomycin for $3 \mathrm{~h}$ were used as apoptotic positive controls as actinomycin (Beijing Solarbio Science \& Technology Co., Ltd.) represses transcription and induce necrosis; untreated cells were used as negative controls. Levels of apoptosis were measured using the Annexin V-fluorescein isothiocyanate (FITC) apoptosis detection kit (NeoBioscience Technology Co., Ltd.) and a flow cytometer. Cells $\left(5 \times 10^{5} / \mathrm{ml}\right)$ were resuspended in $1 \mathrm{X}$ binding buffer and stained with Annexin V-FITC and propidium iodide for $5 \mathrm{~min}$ at room temperature, followed by flow cytometry detection. The rate of apoptosis was analysed using CellQuest software (version 3.3; BD Biosciences).

Assessment of fibulin-5 interference and overexpression on aldosterone-induced apoptosis. Cells overexpressing, knockdown and control cells in the logarithmic growth phase were seeded into a 6 -well plate $\left(5 \times 10^{5}\right.$ cells/well) following overnight incubation were treated with different concentrations of aldosterone for $48 \mathrm{~h}$. Cells were grown under a humidified atmosphere containing $5 \% \mathrm{CO}_{2}$ at $37^{\circ} \mathrm{C}$. Cells were collected and levels of apoptosis were measured using flow cytometry. Cells treated with actinomycin for $3 \mathrm{~h}$ were used as apoptotic positive controls and untreated cells were used as negative controls. 
Statistical analysis. Results were plotted using Graph Pad Prism 6.01 (GraphPad Software, Inc.) and analyzed using one-way analysis of variance followed by a Student-Newman-Keuls test. Data were analysed using the SPSS 21.0 statistical software package (IBM Corp.) and all data are presented as the mean \pm standard deviation of three independent experiment.

\section{Results}

Determination of fibulin-5 interference and overexpression in stable cell lines. The fibulin-5 overexpression and interference lentiviruses were used to infect HA-VSMC cells. Cells were then screened with puromycin for 2 weeks, subsequently mRNA and protein were extracted from cells for analysis. The expression of fibulin-5 mRNA and protein in each treated group was detected by RT-qPCR and western blotting, respectively. The results demonstrated that the expression fibulin-5 in cell lines overexpressing fibulin-5 was significantly higher ( $\sim 3$ times) compared with the control groups and the expression of mRNA in shFibulin-5 interference cells was significantly downregulated by $69 \%$ (Fig. 1). Furthermore, the results of western blotting confirmed that the expression of fibulin-5 protein in cells transfected with overexpressing lentivirus was significantly increased (2 times) compared with control cells (Fig. 2). However, the expression of fibulin-5 in cells transfected with shFibulin-5 was significantly downregulated by $\sim 80 \%$ compared with the control group. These results confirm that stable cell lines exhibiting fibulin-5 overexpression and inhibition were successfully constructed and that they could be used in subsequent experiments.

Aldosterone induces the apoptosis of HA-VSMCs. The effect of different concentrations of aldosterone $(1,10,100$ and $1,000 \mu \mathrm{M})$ on the apoptosis of HA-VSMCs at different time points ( $48 \mathrm{~h}$ and 5 days) was determined using the Annexin V-FITC/propidium iodide Apoptosis Detection kit. The results indicated that treatment with $<10 \mu \mathrm{M}$ aldosterone for 5 days did not induce apoptosis in HA-VSMC cells (Fig. 3). The effect on the apoptosis of HA-VSMCs following treatment with 1,000 $\mu \mathrm{M}$ aldosterone for $24 \mathrm{~h}$ was not significant (data not shown); however, $48 \mathrm{~h}$ treatment with $1,000 \mu \mathrm{M}$ aldosterone was effective at inducing apoptosis. After $72 \mathrm{~h}$ aldosterone treatment, the toxicity was more notable, where the number of C-+ cells increased markedly. Increased apoptotic rate in $\mathrm{C}+-\mathrm{IC}++$ cells was also observed (Fig. 3), in accordance with the results of previous studies $(14,15)$.

Effect of fibulin-5 on the aldosterone-induced apoptosis of $H A-V S M C$. Under the same conditions (logarithmic growth phase and fibulin-5 interference and overexpression), stable strains and the corresponding control cell group were treated with 100 and $1,000 \mu \mathrm{M}$ aldosterone for $48 \mathrm{~h}$. The apoptosis rate of each group was detected by flow cytometry. As presented in Fig. 4, the rates of apoptosis were significantly increased following treatment with high concentrations of aldosterone $(1,000 \mu \mathrm{M})$ in fibulin-5 knockdown cells compared with their respective control. However, fibulin-5 knockdown cells were significantly increased compared with their respective cells following treatment with $100 \mu \mathrm{M}$ Aldo. The rate of apoptosis

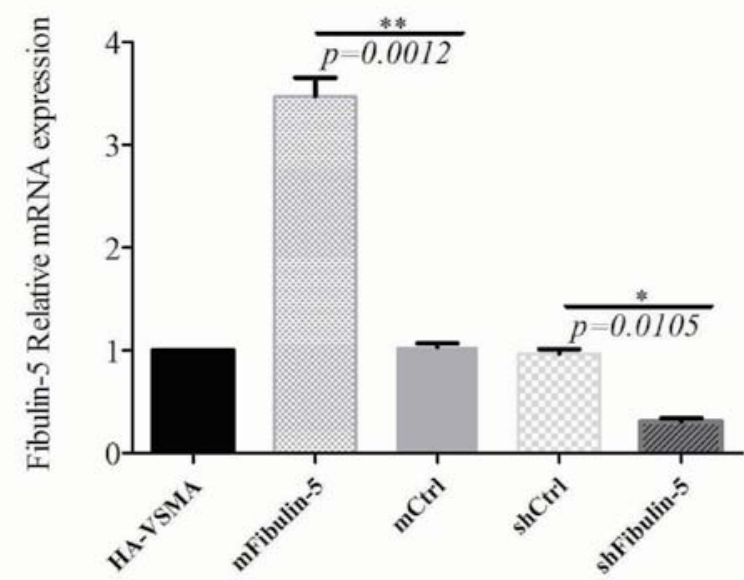

Figure 1. The expression of fibulin-5 mRNA in each treated group was detected by reverse transcription-quantitative polymerase chain reaction. ${ }^{*} \mathrm{P}<0.05,{ }^{* *} \mathrm{P}<0.01$. HA-VSMC, human ascending aortic smooth muscle cells; $\mathrm{Ctrl}$, control; $\mathrm{m}$, magnifying expression vector; sh, short hairpin.

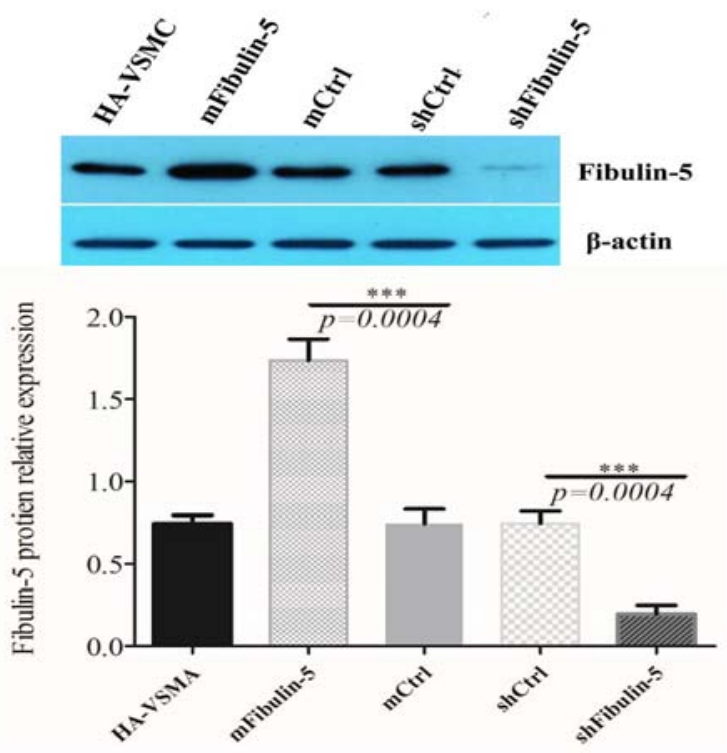

Figure 2. The expression of fibulin-5/ $\beta$-actin protein in the different treatment groups was determined by western blotting. ${ }^{* * *} \mathrm{P}<0.005$. HA-VSMC, human ascending aortic smooth muscle cells; Ctrl, control; $\mathrm{m}$, magnifying expression vector; sh, short hairpin.

in cells overexpressing fibulin-5 significantly decreased following stimulation with low concentrations of aldosterone compared with their respective controls.

These results suggest that fibulin-5 inhibits aldosterone-induced apoptosis. When the aldosterone concentration is high, the inhibitory activity of fibulin-5 is saturated, thereby reducing its inhibitory activity.

\section{Discussion}

PA is the most common cause of secondary hypertension. It was previously thought that patients with PA only accounted for $1-2 \%$ of the total population with hypertension but it has 


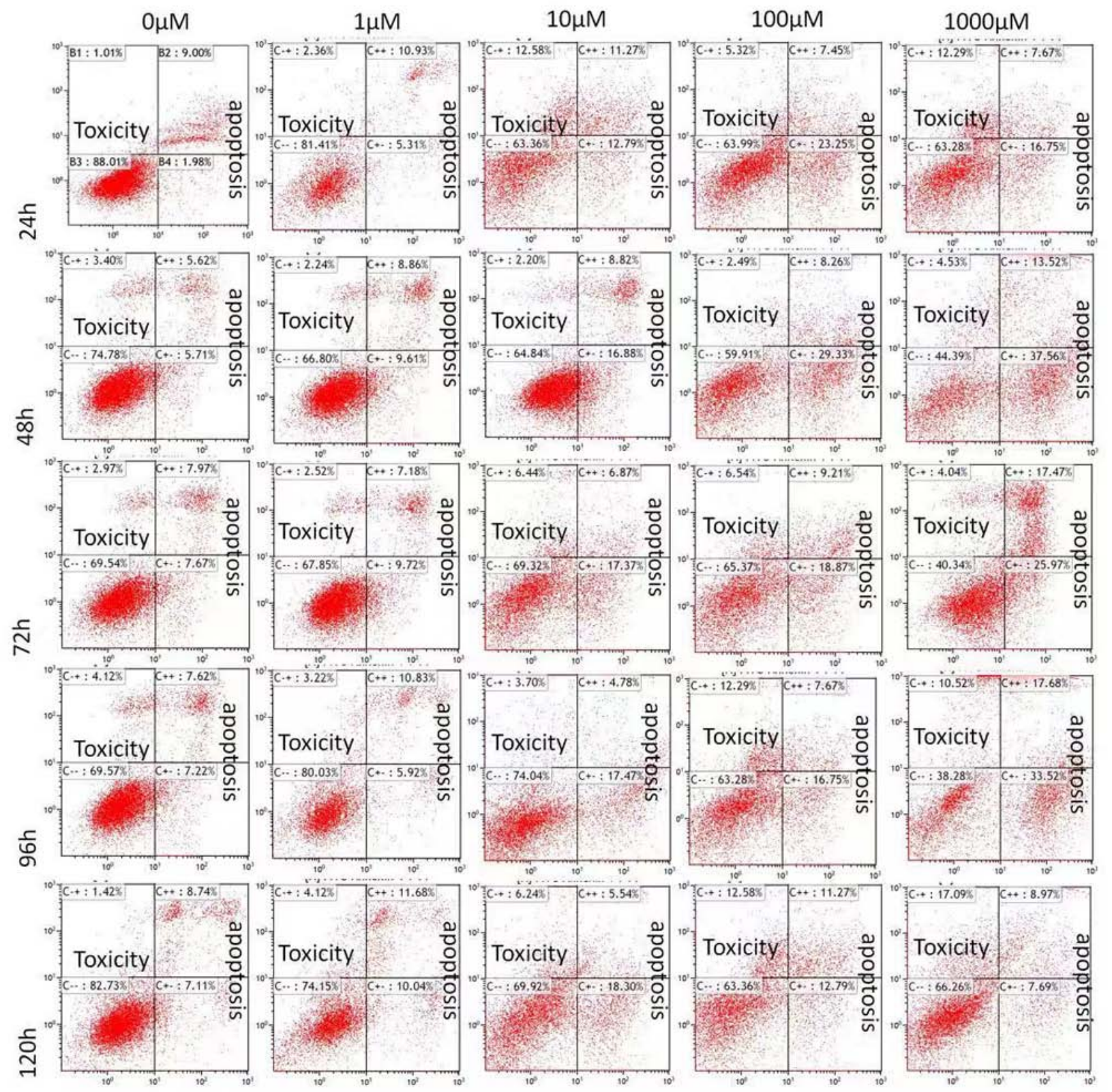

Figure 3. HA-VSMCs were treated with different concentrations of Aldo $(0,1,10,100$ and 1,000 $\mu \mathrm{M})$ for different durations $(1,2,3,4$ and 5 days). Apoptosis was measured using the Annexin V-fluorescein isothiocyanate/propidium iodide Apoptosis Detection kit. HA-VSMC, human ascending aortic smooth muscle cells; Aldo, aldosterone; B1, B2, B3 and B4, normal cell control groups; C-+, dead cells; C++: Late apoptotic cells; C--, living cells; C+-, early apoptotic cells.

been demonstrated that patients with PA account for $>10 \%$ of the total hypertensive population (16). APA is the most common type of PA and accounts for $70-80 \%$ of all cases of PA. The primary method of treating APA is via surgical resection; however, a long-term follow-up study revealed that the postoperative hypertension control rate was only $33-87 \%$ and has become a long-term clinical problem. Vascular remodelling may be responsible for stimulating hypertension; however, the specific mechanism remains to be elucidated.

Aldosterone directly stimulates vascular remodelling and a number of previous studies have indicated that aldosterone damages the cardiovascular system (17-19). Blood vessels can express the mineralocorticoid receptor (MR) and 11ß-hydroxysteroid dehydrogenases (20). The latter is an enzyme that ensures that aldosterone is specifically bound to
MR. Aldosterone can lead to vasoconstriction and elevate blood pressure (21). Hypertension can damage and remodel blood vessels (21). In a previous study, to identify the pathogenesis of PA, a micro-osmotic pump (ALZET 2004) was injected to pump aldosterone into rats and thus establish an animal model of PA. The systolic blood pressure of rats increased, plasma aldosterone concentration increased and renin activity was inhibited following 3 weeks perfusion with aldosterone. After 8 weeks, the mesenteric arteries of the rats exhibited marked changed, including wall thickening and a smaller lumen (3).

Aldosterone induced the apoptosis of VSMCs. Apoptosis serves an important role in the pathogenesis of vascular lesions and excessive apoptosis leads to the loss of vascular cells (18). Previous studies have demonstrated that aldosterone is able induce apoptosis in cardiomyocytes and renal tubular epithelial 
A

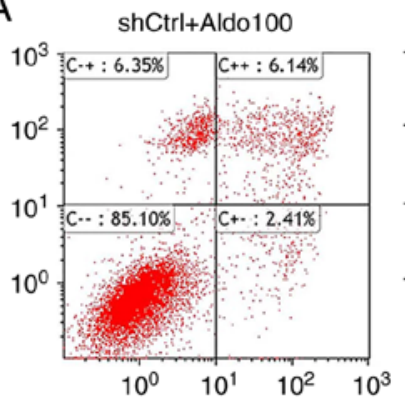

shFibulin-5+Aldo100

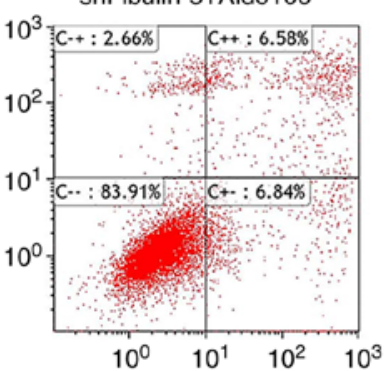

shCtrl+Aldo 1000

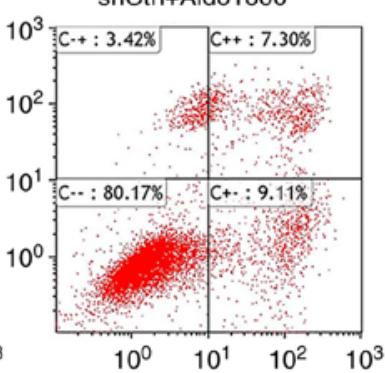

shFibulin-5+Aldo1000

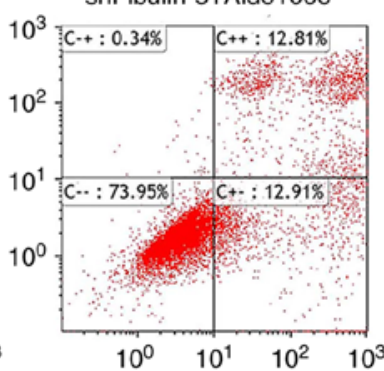

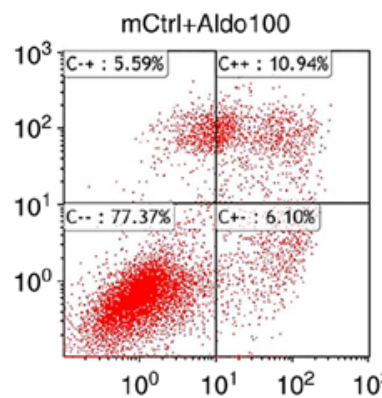
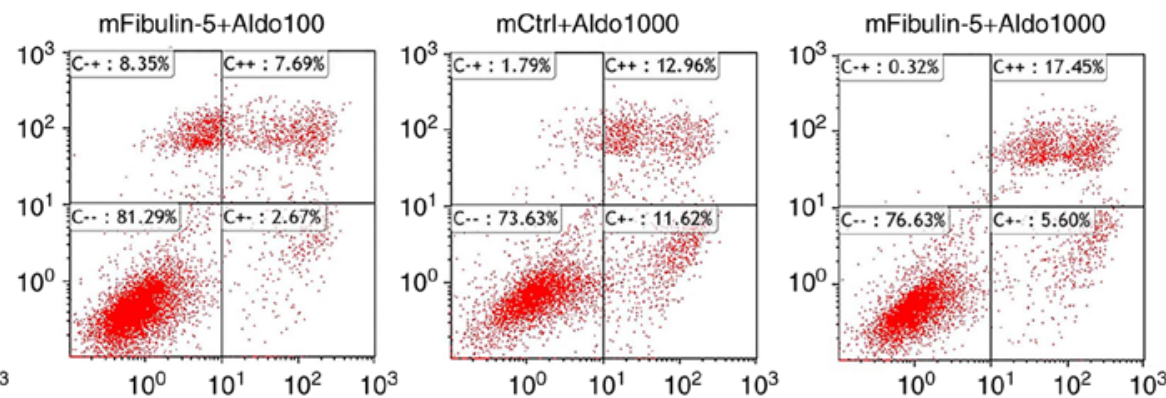

B

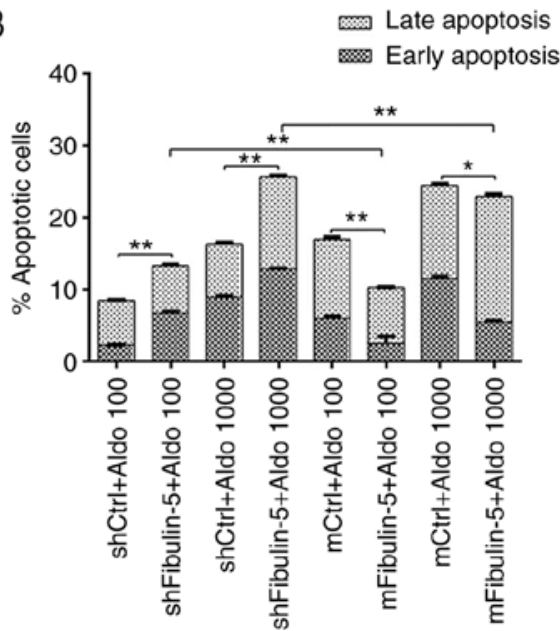

Figure 4. (A) Fibulin-5 inhibited aldosterone-induced apoptosis in HA-VSMCs. (B) Quantification of apoptosis in HA-VSMCs following fibulin-5 overexpression or knockout, and treatment with Aldo. Paired t-tests were performed to evaluate whether differences were significant between groups. Results are expressed as the mean \pm standard deviation. ${ }^{*} \mathrm{P} \leq 0.05$ and ${ }^{* *} \mathrm{P} \leq 0.01$. Aldo, aldosterone; HA-VSMC, human ascending aortic smooth muscle cells; Ctrl, control; $\mathrm{m}$, magnifying expression vector; sh, short hairpin.

cells $(7,17)$. A previous study by the current authors identified found that treatment with aldosterone increased the number of TUNEL-positive VSMCs in the aorta increased, indicating that aldosterone may induce the apoptosis of rat aorta VSMCs in vivo. Furthermore, the expression of the pro-apoptotic proteins Bax, cytochrome $c$ and caspase-3 increased and the expression of the apoptosis inhibition protein Bcl-2 decreased (3). These results indicate that aldosterone induces the apoptosis of VSMCs via the mitochondrial pathway; however, the specific mechanism of action requires further clarification.

Aldosterone induces the deposition of fibulin-5 in the aorta. Extracellular matrix proteins are an important component of vascular remodelling. Fibulin-5 is a member of the fibulin family and is also an important component of the extracellular matrix. It has been demonstrated that fibulin-5 deposition increases in PA rat aortic extracellular matrix proteins (7).
Fibulin-5 is a multifunctional protein that serves an important role in cell proliferation, migration and apoptosis $(4,5)$. The ligation of the carotid artery of fibulin-5 knockout mice is accompanied by severe neointima formation and thickening of the outer membrane (6). The results of a previous study indicated that the deposition of fibulin-5 in the aorta of the rats significantly increased following treatment with aldosterone, suggesting that fibulin-5 may serve an important role in aldosterone-induced vascular remodelling (7).

Fibulin-5 also affects apoptosis. Fibulin-5 has cell- and environment-specific functions; it is able to stimulate the synthesis of fibroblast DNA but also inhibit the synthesis of epithelial cells (8). Fibulin-5 knockout mice exhibited increased intravascular vascular density in normal tissues and reduced endothelial cell apoptosis (9). A polyvinylchloride sponge increased the invasion of new blood vessels in fibulin-5 
knockout mice demonstrating that fibulin-5 inhibited cell invasion (10). Fibrosarcoma cells overexpressing fibulin-5 that were subcutaneously injected into mice inhibited the growth of tumors, suggesting that fibulin-5 promotes apoptosis (11). Under hypoxic conditions, knockout of the fibulin-5 gene significantly increased the apoptosis of endothelial cells (12). The apoptosis of vascular endothelial cells in fibulin-5 knockout mice with pancreatic cancer increases, indicating that fibulin-5 inhibits the apoptosis of endothelial cells (9). Thus, the regulation of apoptosis by fibulin- 5 is different in different types of cells or in different environments. The results of the current study demonstrated that the overexpression of fibulin-5 inhibited aldosterone-induced apoptosis, particularly at lower concentrations of aldosterone and promoted aldosterone-induced apoptosis following fibulin-5 knockdown, particularly at higher concentrations of aldosterone. These results suggest that fibulin-5 inhibits aldosterone-induced $\mathrm{H}-\mathrm{VSMC}$ apoptosis, and this effect may be associated with concentrations of aldosterone in the body.

In conclusion, the results of the present study indicate that fibulin-5 serves a role in the inhibition of aldosterone-induced apoptosis of human aortic smooth muscle cells. The effect of fibulin-5 in vivo may be associated with levels of aldosterone in the body. However, the specific mechanism by which fibulin-5 inhibits apoptosis remains unclear and further studies are required.

\section{Acknowledgements}

Not applicable.

\section{Funding}

The current study was supported by the National Natural Science Foundation of China (grant no. 81360126) and Joint Special Fund of Kunming Medical University (grant no. 2013FB163).

\section{Availability of data and materials}

All data generated or analyzed during this study are included in this published article.

\section{Authors' contributions}

JP and XQN were responsible for the application and design of the whole project, YJY, XJL and XCL jointly completed the experiments, where YJY was responsible for the research direction of the project. XJL and XCL performed vector design, cell transfection and apoptosis measurements. XZ, CZY, and JTT performed cell culture, reagent preparation, statistical analysis and literature research. All authors read and approved the final manuscript.

\section{Ethics approval and consent to participate}

Not applicable.

\section{Patient consent for publication}

Not applicable.

\section{Competing interests}

The authors declare that they have no competing interests.

\section{References}

1. Chen HZ, Zhong NS, Lu ZY, et al: The eighth rdition Textbook of Surgery[M]// published by People's Education Press: Saunders, 2013:708-709. Primary aldosteronism is characterized by the overproduction of the mineralocorticoid hormone aldosterone by the adrenal glands, adrenal cortex hyperplasia or adrenal carcinoma main cause the disease; Symptoms: Occasional muscular weakness, muscle spasms, tingling sensations, or excessive urination the prevalence of APA in hypertension is 10 percent, and the symptom and incidence is like PA.

2. Rossi GP, Bolognesi M, Rizzoni D, Seccia TM, Piva A, Porteri E, Tiberio GA, Giulini SM, Agabiti-Rosei E and Pessina AC: Vascular remodeling and duration of hypertension predict outcome of adrenalectomy in primary aldosteronism patients. Hypertension 51: 1366-1371, 2008.

3. Yan Y, Ouyang J, Wang C, Wu Z, Ma X, Li H, Xu H, Hu Z, Li J, Wang B, et al: Aortic cell apoptosis in rat primary aldosteronism model. J Huazhong Univ Sci Technolog Med Sci 30: 385-390, 2010.

4. Xiao W, Zhou S, Xu H, Li H, He G, Liu Y and Qi Y: Nogo-B promotes the epithelial-mesenchymal transition in HeLa cervical cancer cells via Fibulin-5. Oncol Rep 29: 109-116, 2013.

5. Kapustin A, Stepanova V, Aniol N, Cines DB, Poliakov A, Yarovoi S, Lebedeva T, Wait R, Ryzhakov G, Parfyonova Y, et al: Fibulin-5 binds urokinase-type plasminogen activator and mediates urokinase-stimulated $\beta 1$-integrin-dependent cell migration. Biochem J 443: 491-503, 2012.

6. Spencer JA, Hacker SL, Davis EC, Mecham RP, Knutsen RH, Li DY, Gerard RD, Richardson JA, Olson EN and Yanagisawa H: Altered vascular remodeling in fibulin-5-deficient mice reveals a role of fibulin-5 in smooth muscle cell proliferation and migration. Proc Natl Acad Sci USA 102: 2946-2951, 2005.

7. Ding W, Yang L, Zhang M and Gu Y: Reactive oxygen species-mediated endoplasmic reticulum stress contributes to aldosterone-induced apoptosis in tubular epithelial cells. Biochem Biophys Res Commun 418: 451-456, 2012.

8. Schiemann WP, Blobe GC, Kalume DE, Pandey A and Lodish HF: Context-specific effects of fibulin-5 (DANCE/EVEC) on cell proliferation, motility, and invasion. Fibulin-5 is induced by transforming growth factor-beta and affects protein kinase cascades. J Biol Chem 277: 27367-27377, 2002.

9. Schluterman MK, Chapman SL, Korpanty G, Ozumi K, Fukai T, Yanagisawa $\mathrm{H}$ and Brekken RA: Loss of fibulin-5 binding to betal integrins inhibits tumor growth by increasing the level of ROS. Dis Model Mech 3: 333-342, 2010.

10. Sullivan KM, Bissonnette R, Yanagisawa H, Hussain SN and Davis EC: Fibulin-5 functions as an endogenous angiogenesis inhibitor. Lab Invest 87: 818-827, 2007.

11. Albig AR, Neil JR and Schiemann WP: Fibulins 3 and 5 antagonize tumor angiogenesis in vivo. Cancer Res 66: 2621-2629, 2006.

12. Guadall A, Orriols M, Rodríguez-Calvo R, Calvayrac O, Crespo J, Aledo R, Martínez-González J and Rodríguez C: Fibulin-5 is up-regulated by hypoxia in endothelial cells through a hypoxia-inducible factor-1 (HIF-1 $\alpha$ )-dependent mechanism. J Biol Chem 286: 7093-7103, 2011

13. Livak KJ and Schmittgen TD: Analysis of relative gene expression data using real-time quantitative PCR and the 2(-Delta Delta C(T)) method. Methods 25: 402-408, 2001.

14. Qiao W, Zhang W, Shao S, Gai Y and Zhang M: Effect and mechanism of poly (ADP-ribose) polymerase-1 in aldosterone-induced apoptosis. Mol Med Rep 12: 1631-1638, 2015.

15. Ishizawa K, Izawa Y, Ito H, Miki C, Miyata K, Fujita Y, Kanematsu Y, Tsuchiya K, Tamaki T, Nishiyama A and Yoshizumi M: Aldosterone stimulates vascular smooth muscle cell proliferation via big mitogen-activated protein kinase 1 activation. Hypertension 46: 1046-1052, 2005.

16. Young WF Jr: Minireview: Primary aldosteronism-changing concepts in diagnosis and treatment. Endocrinology 144: 2208-2213, 2003.

17. Hayashi H, Kobara M, Abe M, Tanaka N, Gouda E, Toba H, Yamada H, Tatsumi T, Nakata T and Matsubara H: Aldosterone nongenomically produces NADPH oxidase-dependent reactive oxygen species and induces myocyte apoptosis. Hypertens Res 31: 363-375, 2008. 
18. Korshunov VA and Berk BC: Smooth muscle apoptosis and vascular remodeling. Curr Opin Hematol 15: 250-254, 2008.

19. CalvierL,Miana M,ReboulP,Cachofeiro V,Martinez-MartinezE, de Boer RA, Poirier F, Lacolley P, Zannad F, Rossignol P and López-Andrés N: Galectin-3 mediates aldosterone-induced vascular fibrosis. Arterioscler Thromb Vasc Biol 33: 67-75, 2013.

20. Takeda Y, Miyamori I, Yoneda T, Iki K, Hatakeyama H, Blair IA, Hsieh FY and Takeda R: Production of aldosterone in isolated rat blood vessels. Hypertension 25: 170-173, 1995.
21. Leopold JA: Aldosterone, mineralocorticoid receptor activation, and cardiovascular remodeling. Circulation 124: e466-e468, 2011.

(c) () (5) This work is licensed under a Creative Commons Attribution 4.0 International (CC BY-NC 4.0) License 